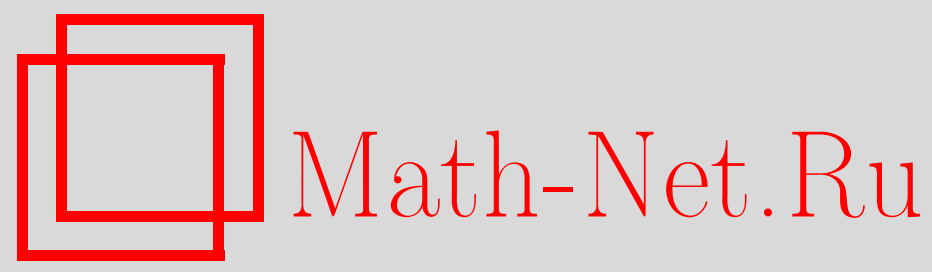

В. Б. Поплавский, О рангах, классах Грина и теории определителей булевых матриц, Дискрет. матем., 2008, том 20, выпуск 4, 42-60

DOI: https://doi.org/10.4213/dm1025

Использование Общероссийского математического портала Math-Net.Ru подразумевает, что вы прочитали и согласны с пользовательским соглашением http://www . mathnet.ru/rus/agreement

Параметры загрузки:

IP: 54.162 .27 .143

26 апреля 2023 г., 13:02:37 
УДК 512.643

\title{
О рангах, классах Грина и теории определителей булевых матриц
}

\author{
() 2008 г. В. Б. Поплавский
}

\begin{abstract}
Рассматривается группоид всевозможных матриц над произвольной булевой алгеброй с частичной операцией произведения матриц. На этом группоиде определяются классы эквивалентностей, аналогичные классам Грина $H, C, R, D, J$ для полугрупп. Вводится понятие минорного ранга булевой матрицы. Показывается, что столбцовые, строчные, факторизационные и минорные ранги являются инвариантами для $J$-класса этого группоида, причем минорные ранги не превосходят столбцовые, строчные, факторизационные и перманентные ранги.

Основной результат статьи объясняет роль булева определителя. Показано, что в некотором $J$-классе существует квадратная $n \times n$ матрица с ненулевым определителем тогда и только тогда, когда столбцовые, строчные, факторизационный и минорный ранги любой матрицы такого класса равны между собой и равны $n$. Bсе $n \times n$ матрицы этого $J$-класса имеют равные определители, а определители квадратных матриц большего размера равны нулю.
\end{abstract}

\section{1. Введение}

В статье продолжается построение теории определителей матриц с элементами из произвольной булевой алгебры и поиск ее приложений. Начатое, по-видимому, в [1], построение теории определителей таких матриц получило свое развитие в работах [2-9].

Определитель квадратной матрицы с элементами из произвольной булевой алгебры определяется как симметрическая разность $\operatorname{Det} A=(\stackrel{+}{\nabla} A \backslash \bar{\nabla} A) \cup(\bar{\nabla} A \backslash \stackrel{+}{\nabla} A)$ полуперманентов

$$
\begin{aligned}
& \stackrel{+}{\nabla} A=\bigcup_{\left(\alpha_{1}, \ldots, \alpha_{n}\right) \in{ }_{P}}\left(a_{1}^{\alpha_{1}} \cap \ldots \cap a_{n}^{\alpha_{n}}\right), \\
& \bar{\nabla} A=\bigcup_{\left(\alpha_{1}, \ldots, \alpha_{n}\right) \in \bar{P}}\left(a_{1}^{\alpha_{1}} \cap \ldots \cap a_{n}^{\alpha_{n}}\right)
\end{aligned}
$$

матрицы $A=\left(a_{j}^{i}\right)$, элементы которой $a_{j}^{i}$ принадлежат произвольной булевой алгебре $\left\langle\mathbf{B}, \cup, \cap,{ }^{\prime}, 0,1\right\rangle$. В этих формулах $\stackrel{+}{P}$ и $\bar{P}$ обозначают соответственно все четные и нечетные перестановки верхних строчных индексов.

Известны такие свойства определителя, как сохранение при транспонировании, неизменность при перестановке строк, свойство обращаться в ноль, если строки (столбцы) матрицы линейно зависимы (обратное не выполняется в общем случае), см. [1]. Известно 
также, что полуперманенты раскладываются по строке или по столбцу по правилу Лаплаca, а определители - не всегда, см. [2-5]. Далее в разделе 5 этой статьи приводятся аналоги формул Коши-Бине для определителей и перманентов произведений булевых матриц. Вывод опирается на соответствующие формулы Коши-Бине для полуперманентов, которые можно найти в [3] и в одновременно вышедших статьях [4, 9]. Здесь же мы доказываем еще одно свойство определителя (предложение 12), обобщающее свойство определителя обращаться в ноль, если строки (столбцы) матрицы линейно зависимы. Предложение 12 играет важную роль для введенного в этой статье (в разделе 6) понятия минорного ранга и помогает оценить число образующих, порождающих своими линейными комбинациями столбцы (или строки) данной булевой матрицы.

Соотношения между различными ранговыми функциями для матриц над полукольцами, а также изменение их значений относительно операций с матрицами хорошо изучены, см. [10-14]. Мы показываем, что введенный здесь минорный ранг булевой матрицы не превосходит факторизационный, а также строчные, столбцовые и перманентный (граничный) ранги данной матрицы (теоремы 2 и 4). Доказывается также, что минорный ранг произведения любых булевых матриц не превосходит минорных рангов сомножителей (теорема 5).

В разделе 4 рассматривается группоид всевозможных булевых матриц конечного размера с частичной операцией произведения и на нем проводится построение классов эквивалентностей $\varepsilon_{H}, \varepsilon_{C}, \varepsilon_{R}, \varepsilon_{D}, \varepsilon_{J}$, аналогичных классам $H, R, C, D, J$ Грина для полугрупп соответственно, см. гл. 2 в [15] и гл. 2 в [16]. Далее в работе показывается, что определители всех квадратных матриц одного и того же размера равны, если они находятся в одном таком классе $\varepsilon_{J}$ (аналоге $J$-класса) группоида всевозможных булевых матриц конечного размера (теорема 3). Доказывается также, что факторизационные и минорные ранги, а также размерности строчных и столбцовых пространств матриц, являются инвариантами для такого $\varepsilon_{J}$-класса группоида.

Основной результат этой статьи, проясняющий роль определителя, сформулирован в теореме 6 и заключается в том, что в некотором $\varepsilon J$-классе существует квадратная $n \times n$ матрица с ненулевым определителем тогда и только тогда, когда размерности строчного и столбцового пространства, факторизационный и минорный ранги любой матрицы такого класса равны между собой и равны $n$. Все матрицы того же размера $n \times n$ имеют равные определители, а определители квадратных матриц этого $\varepsilon$-класса большего размера, чем $n$, равны нулю.

\section{2. Алгебры булевых матриц}

Определение 1. Булевой алгеброй называют систему $\left\langle B, \cup, \cap,{ }^{\prime}, 0,1\right\rangle$, где $B$ - множество (носитель), $\cup: B \times B \rightarrow B, \cap: B \times B \rightarrow B-$ две бинарные операции, ': $B \rightarrow B-$ унарная операция, а 0 и $1-$ различные $(0 \neq 1)$ элементы из $B$ такие, что следующие тождества выполняются для любых $x, y, z \in B$ :

1.1. $x \cup y=y \cup x$,

1.2. $x \cap y=y \cap x$,

2.1. $(x \cup y) \cup z=x \cup(y \cup z)$,

2.2. $(x \cap y) \cap z=x \cap(y \cap z)$,

3.1. $x \cup(x \cap y)=x$, 
3.2. $x \cap(x \cup y)=x$,

4.1. $x \cup(y \cap z)=(x \cup y) \cap(x \cup z)$,

4.2. $x \cap(y \cup z)=(x \cap y) \cup(x \cap z)$,

5.1. $x \cap 0=0$,

5.2. $x \cup 1=1$,

6.1. $x \cup x^{\prime}=1$,

6.2. $x \cap x^{\prime}=0$.

Назовем $\cup$ объединением, $\cap$ пересечением, a ' дополнением. Таким образом, объединение и пересечение - коммутативные (1.1 и 1.2), ассоциативные (2.1 и 2.2), удовлетворяющие законам поглощения (3.1 и 3.2) операции. Это означает, что $\langle B, \cup, \cap\rangle$ есть решетка. Эта решетка дистрибутивна (4.1 и 4.2), обладает нулем (5.1) и единицей (5.2). Более того, эта решетка есть решетка с дополнением (6.1 и 6.2).

Ясно, что матрицы одного и того же размера $m \times n$ с элементами из произвольной булевой алгебры $\left\langle\mathbf{B}, \cup, \cap,{ }^{\prime}, 0,1\right\rangle$ образуют булеву алгебру $\left\langle\mathbf{B}_{m \times n}, \cup, \cap,{ }^{\prime}, O, J\right\rangle$, операции объединения $\cup$, пересечения $\cap$ и дополнения ' матриц (следовательно, и отношение частичного порядка) определяются поэлементно. Нулем и единицей такой вторичной булевой алгебры служат матрицы $O$ и $J$ размера $m \times n$, образованные целиком из нулей 0 и единиц 1 соответственно.

С другой стороны, булевы матрицы одного и того же размера из $\mathbf{B}_{m \times n}$ образуют полукольцевое векторное пространство, то есть непустое множество с двумя операциями, объединением матриц $A \cup B=\left(a_{j}^{i} \cup b_{j}^{i}\right) \in \mathbf{B}_{m \times n}$ (заменяющим сложение) и пересечением матрицы с элементом из булевой алгебры $\lambda \cap A=\left(\lambda \cap a_{j}^{i}\right) \in \mathbf{B}_{m \times n}$ (заменяющим умножение на скаляр), удовлетворяющими для любых матриц и булевых скаляров следующим аксиомам:

(1) $(A \cup B) \cup C=A \cup(B \cup C)$,

(2) $A \cup B=B \cup A$,

(3) $1 \cap A=A$,

(4) $(\alpha \cap \beta) \cap A=\alpha \cap(\beta \cap A)$,

(5) $(\alpha \cup \beta) \cap A=(\alpha \cap A) \cup(\beta \cap A)$,

(6) $\alpha \cap(A \cup B)=(\alpha \cap A) \cup(\alpha \cap B)$.

Это позволяет далее говорить о линейных комбинациях булевых матриц, вводить понятия линейной зависимости и независимости матриц.

Особое внимание в этой работе уделяется множеству всевозможных матриц с одной частичной операцией, определяемой далее.

Определение 2. Определим конъюнктное произведение $m \times n$ матрицы $A=\left(a_{j}^{i}\right)$ из $\mathbf{B}_{m \times n}$ на $n \times k$ матрицу $B=\left(b_{s}^{t}\right)$ из $\mathbf{B}_{n \times k}$ как матрицу $C=A \sqcap B$ размера $m \times k$, элементы $c_{s}^{i}$ которой вычисляются по формуле $c_{s}^{i}=\bigcup_{t=1}^{n}\left(a_{t}^{i} \cap b_{s}^{t}\right)$. 
Дуальным образом можно определить дизъюнктное произведение матриц $C=A \sqcup B$ как матрицу, элементы которой вычисляются по формуле $c_{s}^{i}=\bigcap_{t=1}^{n}\left(a_{t}^{i} \cup b_{s}^{t}\right)$. Однако, так как $(A \sqcap B)^{\prime}=A^{\prime} \sqcup B^{\prime}$ и далее будет рассматриваться только конъюнктное произведение, именно конъюнктное произведение будем иметь в виду под термином произведение.

Введенное произведение обладает следующими свойствами, выполняющимися для произвольных матриц $A, B, C$ соответствующих размеров и произвольных булевых скаляров $\lambda \in \mathbf{B}$ :

(1) $A \sqcap(B \sqcap C)=(A \sqcap B) \sqcap C)$,

(2) $A \sqcap(B \cup C)=(A \sqcap B) \cup(A \sqcap C),(A \cup B) \sqcap C=(A \sqcap C) \cup(B \sqcap C)$,

(3) $A \sqcap E=A, E \sqcap A=A$,

(4) $A \sqcap O=O, O \sqcap A=O$,

(5) $(A \sqcap B)^{T}=B^{T} \sqcap A^{T}$,

(6) $A \sqcap(B \cap C) \subseteq(A \sqcap B) \cap(A \sqcap C),(A \cap B) \sqcap C \subseteq(A \sqcap C) \cap(B \sqcap C)$,

(7) $\lambda \cap(A \sqcap B)=(\lambda \cap A) \sqcap B=A \sqcap(\lambda \cap B)=(\lambda \cap A) \sqcap(\lambda \cap B)$,

(8) $\lambda \cup(A \sqcap B)=(\lambda \cup A) \sqcap(\lambda \cup B)$.

Здесь $E=\left(\delta_{j}^{i}\right)$ есть единичная матрица соответствующего размера $\left(\delta_{j}^{i}\right.$ принимает значение 1 , если $i=j$, и значение 0 , если $i \neq j$ ), $O$ есть нулевая матрица соответствующего размера, $A^{T}$ означает транспонирование матрицы $A$.

Легко видеть, что из включения $A \subseteq B$ следует включение $D \sqcap A \subseteq D \sqcap B$, поэтому множество всевозможных булевых матриц относительно произведения образует решеточно упорядоченный группоид $\mathbf{M}(\mathbf{B})$ относительно ассоциативной частичной операции произведения П.

\section{3. Строчные, столбцовые и факторизационные ранги булевой матрицы}

Определение 3. Линейной комбинацией $m \times n$ матриц $B_{1}, \ldots, B_{k}$ с коэффициентами $\lambda^{1}, \ldots, \lambda^{k}$ называется $\left(\lambda^{1} \cap B_{1}\right) \cup \ldots \cup\left(\lambda^{k} \cap B_{k}\right)$. Множество $S\left(\left\{B_{1}, \ldots, B_{k}\right\}\right)$ всевозможных линейных комбинаций некоторых $m \times n$ матриц $B_{1}, \ldots, B_{k}$ назовем линейной оболочкой данных булевых матриц. Матрица $B$ называется зависимой от матриц $B_{1}, \ldots, B_{k}$, если $B \in S\left(\left\{B_{1}, \ldots, B_{k}\right\}\right)$. Множество матриц $B_{1}, \ldots, B_{k}$ назовем множеством независимых матриц, если каждая матрица $B_{j}, j=1, \ldots, k$, не принадлежит линейной оболочке $S\left(\left\{B_{1}, \ldots, B_{j-1}, B_{j+1}, \ldots, B_{k}\right\}\right)$ других матриц этого множества.

Отметим следующие свойства линейных оболочек матриц одного размера.

Предложение 1. Каждая из матрии $B_{1}, \ldots, B_{k}$ зависит от матрии $C_{1}, \ldots, C_{s}$, m.e. является элементом $S\left(\left\{C_{1}, \ldots, C_{s}\right\}\right)$, тогда и только тогда, когда $S\left(\left\{B_{1}, \ldots, B_{k}\right\}\right) \subseteq$ $S\left(\left\{C_{1}, \ldots, C_{s}\right\}\right)$.

Доказательство. Необходимость следует из того, что линейная комбинация линейных комбинаций некоторого множества матриц $C_{1}, \ldots, C_{s}$ является линейной комбинацией этого множества матриц. Достаточность следует из того, что всегда $B_{i} \in S\left(\left\{B_{1}, \ldots, B_{k}\right\}\right)$, $i=1, \ldots, k$. Следовательно, из включения $S\left(\left\{B_{1}, \ldots, B_{k}\right\}\right) \subseteq S\left(\left\{C_{1}, \ldots, C_{s}\right\}\right)$ следует, что $B_{1}, \ldots, B_{k}$ зависят от матриц $C_{1}, \ldots, C_{s}$. 
Следующее утверждение позволяет избавляться в списке матриц, порождающих данную оболочку, от таких матриц, которые зависят от остальных.

Следствие 1. Если $B_{j}$ есть линейная комбинация матрии $B_{1}, \ldots, B_{j-1}, B_{j+1}, \ldots, B_{k}$, то $S\left(\left\{B_{1}, \ldots, B_{j-1}, B_{j}, B_{j+1}, \ldots, B_{k}\right\}\right)=S\left(\left\{B_{1}, \ldots, B_{j-1}, B_{j+1}, \ldots, B_{k}\right\}\right)$.

Доказательство. Каждая матрица из множества $\left\{B_{1}, \ldots, B_{j-1}, B_{j}, B_{j+1}, \ldots, B_{k}\right\}$ является линейной комбинацией матриц множества $\left\{B_{1}, \ldots, B_{j-1}, B_{j+1}, \ldots, B_{k}\right\}$ и наоборот, поэтому из предложения 1 следует данное утверждение.

Определение 4. Обозначим $C(A)$ линейную оболочку всех столбцов $A_{1}, \ldots, A_{n}$ некоторой булевой $m \times n$ матрицы $A$ и назовем ее столбцовым пространством. Строчное пространство $R(A)$ булевой $m \times n$ матрицы $A$ определяется как линейная оболочка всех строк $A^{1}, \ldots, A^{m}$ данной матрицы $A$.

Упорядоченный набор $A_{1}, \ldots, A_{k}$ независимых столбцов некоторой матрицы $A$ такой, что $C(A)=S\left(\left\{A_{1}, \ldots, A_{n}\right\}\right), k \leqslant n$, назовем столбцовым базисом матрицы $A$. Аналогично определяется строчный базис $A^{1}, \ldots, A^{s}, s \leqslant m$.

Множество всех столбцовых базисов (или всех строчных базисов) матрицы $A$ может содержать базисы, состоящие из различного числа столбцов (или строк). Это демонстрируют следующие примеры.

Пример 1. Для булевой матрицы

$$
A=\left(\begin{array}{ccc}
{[1 ; 3]} & {[2 ; 3]} & \varnothing \\
{[3 ; 5]} & {[3 ; 4]} & \{5\} \\
{[1 ; 2]} & \{2\} & \varnothing \\
{[2 ; 4]} & {[2 ; 4]} & \varnothing \\
(4 ; 5] & \varnothing & \{5\}
\end{array}\right)
$$

составленной из подмножеств действительной числовой прямой, первые две и оставшиеся три строчки образуют базисы ее строчного пространства. Столбцовый базис один и состоит из двух первых столбцов этой матрицы.

Пример 2. В матрице

$$
A=\left(\begin{array}{lll}
(11100) & (01100) & (00000) \\
(00111) & (00110) & (00001) \\
(11111) & (01110) & (00001)
\end{array}\right)
$$

построенной над $\mathbf{B}_{2}^{5}-$ пятой степенью двухэлементной булевой алгебры $\mathbf{B}_{2}=\{0,1\}$, можно найти два строчных базиса $b^{1}=\left\{A^{1}, A^{2}\right\}$ и $b^{2}=\left\{A^{3}\right\}$, так как $A^{3}=A^{1} \cup A^{2}$, $A^{1}=(11100) \cap A^{3}$ и $A^{2}=(00111) \cap A^{3}$. Столбцовый базис единствен и состоит из $b_{1}=\left\{A_{1}\right\}$. Действительно, $A_{2}=(01110) \cap A_{1}$ и $A_{3}=(00001) \cap A_{1}$.

Определение 5. Размерностью пространства столбцов $C(A)$ или столбцовым рангом матрицы $A$ назовем число, обозначаемое либо $\operatorname{dim} C(A)$, либо $\operatorname{rank}_{C} A$, и равное наименьшему возможному числу столбцов всех таких матриц $B$, для которых $C(A)=C(B)$. Соответственно, размерностью пространства строк $R(A)$ или строчным рангом матрицы $A$ назовем число, обозначаемое либо $\operatorname{dim} R(A)$, либо $\operatorname{rank}_{R} A$ и равное наименьшему возможному числу строк всех таких матриц $B$, для которых $R(A)=R(B)$. 
Известно, что в общем случае $\operatorname{rank}_{C} A \neq \operatorname{rank}_{R} A$. Из определения 5 следует, что для любой булевой матрицы $A$ существует матрица $B$ с тем же самым столбцовым (строчным) пространством такая, что $\operatorname{rank}_{C} B=\operatorname{rank}_{C} A\left(\operatorname{rank}_{R} B=\operatorname{rank}_{R} A\right)$. Причем, матрицу $B$ можно заведомо считать образованной только из тех столбцов, что дают минимальный столбцовый базис пространства столбцов $C(A)$. Такое же замечание можно сделать и для строк. Так, для матрицы $A$ из примера 1 минимальный строчный базис состоит из одной строчки $B=([1 ; 5] \quad[2 ; 4] \quad\{5\})$, то есть $\operatorname{rank}_{R} A=1$.

В случае двухэлементной булевой алгебры $(0,1)$ матрицы обладают, причем единственным, минимальным столбцовым (или строчным) базисом. Доказательство этого факта можно найти в [14] (см. теорему 1.1.1 в разделе 1.1). По-видимому, это не происходит в общем случае, при выборе других булевых алгебр, над которыми строится матричная алгебра.

Предложение 2. Пусть $A$ и $B$ - булевы матрицы с одинаковым числом строк (или столбцов). Для выполнения равенства $A=B \sqcap U$ (или $A=U \sqcap B$ ), где $U-$ некоторая булева матрица подходящеего размера, необходимо и достаточно, чтобы $C(A) \subseteq C(B)$ (или $R(A) \subseteq R(B))$.

Доказательство. Действительно, если $A=B \sqcap U$ для некоторой булевой матрицы $U$, то, как и для числовых матриц, каждый $i$-й столбец матрицы $A$ есть некоторая линейная комбинация столбцов матрицы $B$ с коэффициентами, составляющими $i$-й столбец матрицы $U$. Используя предложение 1 , получаем, что $C(A) \subseteq C(B)$. Справедливость обратного утверждения доказывается аналогично.

Следующие критерии совпадения столбцовых или строчных пространств булевых матриц непосредственно следуют из предложения 2.

Предложение 3. Две матрицы с одинаковым числом строк (столбцов) $A$ и $B$ имеют одинаковые столбиовые пространства $C(A)=C(B)$ (соответственно $R(A)=R(B)$ ) тогда и только тогда, когда $A=B \sqcap U$ u $B=A \sqcap V$ (соответственно $A=U \sqcap B$ u $B=V \sqcap A)$ для некоторых булевых матриц $U$ u $V$ подходящего размера, то есть порождают один и тот же главный правый (левый) идеал в группоиде М(В).

Предложение 4. Две матрицьь с одинаковым числом строк (или столбцов) $A$ и $B$ имеют одинаковые столбиовые пространства $C(A)=C(B)($ соответственно $R(A)=R(B)$ ) тогда и только тогда, когда для любой матрицы $D$ соответствующего размера $C(D \sqcap A)=C(D \sqcap B)($ соответственно $R(A \sqcap D)=R(B \sqcap D))$.

Доказательство. Из равенств $A=B \sqcap U$ и $B=A \sqcap V$ следует, что $D \sqcap A=D \sqcap B \sqcap U$ и $D \sqcap B=D \sqcap A \sqcap V$. Это и означает, что из равенства $C(A)=C(B)$ следует равенство $C(D \sqcap A)=C(D \sqcap B)$, где $D-$ произвольная матрица соответствующего размера.

Обратно, в силу произвольности матрицы $D$, ее можно взять единичной, тогда из равенства $C(D \sqcap A)=C(D \sqcap B)$ сразу следует, что $C(A)=C(B)$.

Аналогично, из равенства $R(A)=R(B)$ выводится равенство $R(A \sqcap D)=R(B \sqcap D)$ и наоборот.

Определение 6. Пусть $A_{m \times n} \in \mathbf{B}_{m \times n}-$ некоторая булева матрица. Наименьшее число $r$, для которого существуют матрицы $\tilde{A}_{m \times r} \in \mathbf{B}_{m \times r}$ и $\tilde{B}_{r \times n} \in \mathbf{B}_{r \times n}$ такие, что $A_{m \times n}=\tilde{A}_{m \times r} \sqcap \tilde{\tilde{A}}_{r \times n}$, называется рангом факторизации (или факторизационным рангом) матрицы $A_{m \times n}$. Для ранга факторизации введем обозначение $r=\operatorname{rank}_{f} A$. 
Возможен и другой способ определения ранга факторизации, использующий понятие кросс-матрицы или кросс-вектора. Кросс-матрицей называют матрицу, представимую в виде произведения матрицы-столбца на матрицу-строку. Очевидно, минимальное число кросс-матриц, объединение которых дает данную булеву матрицу, есть ее ранг факторизации.

Третья интерпретация ранга факторизации означает, что каждый столбец или строка матрицы $A_{m \times n}$ есть линейная комбинация каких-то, не обязательно принадлежащих самой матрице $A_{m \times n}$, столбцов или строк в числе $r=\operatorname{rank}_{f} A$, и это число нельзя сделать меньше.

Предложение 5. Для любой булевой матрищь справедливы неравенства

$$
\operatorname{rank}_{f} A \leqslant \operatorname{dim} C(A)=\operatorname{rank}_{C} A, \quad \operatorname{rank}_{f} A \leqslant \operatorname{dim}_{R}(A)=\operatorname{rank}_{R} A .
$$

Доказательство. Для матрицы $B_{m \times k}$ такой, что $\operatorname{rank}_{C}\left(B_{m \times k}\right)=\operatorname{dim} C\left(A_{m \times n}\right)=k$, верно равенство $C\left(B_{m \times k}\right)=C\left(A_{m \times n}\right)$, что влечет существование равенств $B_{m \times k}=A_{m \times n} \sqcap U_{n \times k}$ и $A_{m \times n}=B_{m \times k} \sqcap V_{k \times n}$ (см. предложение 3). Тогда $A_{m \times n}=A_{m \times n} \sqcap U_{n \times k} \sqcap V_{k \times n}=$ $W_{m \times k} \sqcap V_{k \times n}$, где $W_{m \times k}=A_{m \times n} \sqcap U_{n \times k}$. Отсюда получаем, что $\operatorname{rank}_{f} A \leqslant k=\operatorname{dim}_{C}(A)$, так как в противном случае это противоречило бы понятию факторизационного ранга.

Аналогично доказывается соотношение $\operatorname{rank}_{f} A \leqslant k=\operatorname{rank}_{R} A$.

Предложение 6. Для любых двух булевых матрии $A, B$ подходящего размера ранг факторизации произведения не превосходит рангов факторизащии сомножителей:

$$
\operatorname{rank}_{f} A \sqcap B \leqslant \operatorname{rank}_{f} A, \quad \operatorname{rank}_{f} A \sqcap B \leqslant \operatorname{rank}_{f} B .
$$

Доказательство. Действительно, пусть $\operatorname{rank}_{f} A=r$ и $A_{m \times n}=\tilde{A}_{m \times r} \sqcap \tilde{\tilde{A}}_{r \times n}$. Тогда булева матрица $C=A \sqcap B$, в силу возможности ее представления в виде $C=A \sqcap B=$ $\tilde{A}_{m \times r} \sqcap \tilde{\tilde{A}}_{r \times n} \sqcap B=\tilde{A}_{m \times r} \sqcap \tilde{\tilde{B}}$, где $\tilde{\tilde{B}}=\tilde{\tilde{A}}_{r \times n} \sqcap B$, не может иметь ранг, больший $r$.

\section{4. Классы Грина группоида М(В)}

Отношения двух матриц $A$ и $B$, для которых столбцовые или строчные пространства одинаковы, очевидно, являются отношениями эквивалентности на ассоциативном частичном группоиде М(В) булевых матриц всевозможных конечных размеров. Обозначим эти отношения символами $\varepsilon_{C}$ и $\varepsilon_{R}$ соответственно. Обозначим

$$
\varepsilon_{C}\langle A\rangle=\{B \in \mathbf{M}(\mathbf{B}): C(B)=C(A)\}, \quad \varepsilon_{R}\langle A\rangle=\{B \in \mathbf{M}(\mathbf{B}): R(B)=R(A)\}
$$

соответствующие классы эквивалентности, порожденные элементом $A$.

Пересечение эквивалентностей $\varepsilon_{H}=\varepsilon_{C} \cap \varepsilon_{R}$, рассматриваемое как пересечение подмножеств квадрата $\mathbf{M}(\mathbf{B}) \times \mathbf{M}(\mathbf{B})$, также является эквивалентностью, ее классы определяются условием

$$
\varepsilon_{R}\langle A\rangle=\{B \in \mathbf{M}(\mathbf{B}):(C(B)=C(A)) \wedge(R(B)=R(A))\}=\varepsilon_{C}\langle A\rangle \cap \varepsilon_{R}\langle A\rangle .
$$

Можно рассмотреть объединение бинарных отношений $\varepsilon_{C} \cup \varepsilon_{R}$. Однако объединение эквивалентностей в общем случае эквивалентностью не является, так как для него не выполняется условие транзитивности. Поэтому берем транзитивное замыкание объединения бинарных отношений

$$
\varepsilon_{D}=\bigcup_{k=1}^{\infty}\left(\varepsilon_{C} \cup \varepsilon_{R}\right)^{k}=\varepsilon_{C} \vee \varepsilon_{R},
$$


как минимальное транзитивное отношение, содержащее $\varepsilon_{C}$ и $\varepsilon_{R}$.

Предложение 7. Справедливы равенства

$$
\varepsilon_{D}=\varepsilon_{C} \vee \varepsilon_{R}=\varepsilon_{C} \cdot \varepsilon_{R}=\varepsilon_{R} \cdot \varepsilon_{C}
$$

Доказательство. Сначала покажем, что $\varepsilon_{C} \cdot \varepsilon_{R}=\varepsilon_{R} \cdot \varepsilon_{C}$. Если $(A, B) \in \varepsilon_{C} \cdot \varepsilon_{R}$, то по определению произведения бинарных отношений это означает существование такой матрицы $D$, что $(A, D) \in \varepsilon_{C}$ и $(D, B) \in \varepsilon_{R}$. Последнее означает, что $C(A)=C(D)$ и $R(D)=R(B)$. Отсюда, с учетом предложения 3, следует, что $A=D \sqcap U$ и $B=V \sqcap D$ для некоторых матриц $U$ и $V$. Это дает равенство $V \sqcap A=V \sqcap D \sqcap U=B \sqcap U$. C другой стороны, с учетом предложения 4, равенства $C(A)=C(D)$ и $R(D)=R(B)$ дают $C(V \sqcap A)=C(V \sqcap D)$ и $R(D \sqcap U)=R(B \sqcap U)$ соответственно. Таким образом, найдена матрица $W=V \sqcap A=B \sqcap U$, для которой $C(W)=C(B)$ и $R(A)=R(W)$. Это означает, что $(A, B) \in \varepsilon_{R} \cdot \varepsilon_{C}$ и поэтому $\varepsilon_{C} \cdot \varepsilon_{R} \subseteq \varepsilon_{R} \cdot \varepsilon_{C}$. Обратное включение доказывается аналогично. Следовательно, $\varepsilon_{C} \cdot \varepsilon_{R}=\varepsilon_{R} \cdot \varepsilon_{C}$.

Известно (см. лемму 1.4 в разделе 1.4 в [15]), что композиция коммутирующих эквивалентностей является эквивалентностью. Поэтому произведения $\varepsilon_{C} \cdot \varepsilon_{R}=\varepsilon_{R} \cdot \varepsilon_{C}$ являются эквивалентностями. Кроме этого, очевидно, что транзитивное замыкание $\bigcup_{k=1}^{\infty}\left(\varepsilon_{C} \cup \varepsilon_{R}\right)^{k}$ объединения $\varepsilon_{C} \cup \varepsilon_{R}$ содержит композиции $\varepsilon_{C} \cdot \varepsilon_{R}=\varepsilon_{R} \cdot \varepsilon_{C}$ и, в силу минимальности, совпадает с ними.

Предложение 8. Два класса $\varepsilon_{C}\langle A\rangle$ u $\varepsilon_{R}\langle B\rangle$ пересекаются тогда и только тогда, когда они содержатся в одном и том же классе отношения $\varepsilon_{D}$.

Доказательство. Пусть $\varepsilon_{C}\langle A\rangle \cap \varepsilon_{R}\langle B\rangle \neq \varnothing$. Это эквивалентно существованию матрицы $X \in \mathbf{M}(\mathbf{B})$ такой, что $X \in \varepsilon_{C}\langle A\rangle$ и $X \in \varepsilon_{R}\langle B\rangle$, то есть $(A, X) \in \varepsilon_{C}$ и $(X, B) \in \varepsilon_{R}$. Последнее означает, что $(A, B) \in \varepsilon_{C} \cdot \varepsilon_{R}=\varepsilon_{D}$.

Предложение 8 дает следующее очевидное следствие: два класса $\varepsilon_{D}\langle A\rangle$ и $\varepsilon_{D}\langle B\rangle$ совпадают тогда и только тогда, когда классы $\varepsilon_{C}\langle A\rangle$ и $\varepsilon_{R}\langle B\rangle$ пересекаются.

Определение 7. Будем говорить, что булевы матрицы $A$ и $B$ из группоида М(B) находятся в отношении $\varepsilon_{J}$, если найдутся матрицы $U_{1}, U_{2}, V_{1}, V_{2}$ подходящих размеров такие, что $A=V_{1} \sqcap B \sqcap U_{1}$ и $B=V_{2} \sqcap A \sqcap U_{2}$.

Очевидно, $\varepsilon_{J}$ является отношением эквивалентности. Кроме того, если $A$ и $B$ находятся в отношении $\varepsilon_{D}$, то классы $\varepsilon_{C}\langle A\rangle$ и $\varepsilon_{R}\langle B\rangle$ пересекаются (предложение 8). Тогда найдется такая матрица $X \in \mathbf{M}(\mathbf{B})$ и такие матрицы $U_{1}, U_{2}, V_{1}, V_{2} \in \mathbf{M}(\mathbf{B})$, что $X \in \varepsilon_{C}\langle A\rangle$ или $A=X \sqcap U_{1}, X=A \sqcap U_{2}$, а также $X \in \varepsilon_{R}\langle B\rangle$ или $B=V_{2} \sqcap X, X=V_{1} \sqcap B$ (предложение 3). Получаем, что $A=V_{1} \sqcap B \sqcap U_{1}$ и $B=V_{2} \sqcap A \sqcap U_{2}$, то есть $\varepsilon_{D} \subseteq \varepsilon_{J}$.

На самом деле, для булевых матриц частичного группоида $\mathbf{M ( B ) ~ в ы п о л н я е т с я ~ с л е д у ю - ~}$ щее утверждение, аналогичное известному результату из теории полугрупп, состоящему в том, что для устойчивых полугрупп, в частности, периодических полугрупп, классы Грина $D$ и $J$ совпадают (см. раздел 3 в гл. 2 в [16]).

Предложение 9. Для булевых матриц частичного группоида отношения эквивалентносmи $\varepsilon_{D}$ и $\varepsilon_{J}$ совпадают.

Доказательство. Из справедливости включения $(A, B) \in \varepsilon_{J}$ по определению следует, что $A=V_{1} \sqcap B \sqcap U_{1}$ и $B=V_{2} \sqcap A \sqcap U_{2}$, что дает возможность представления матрицы $A$ в виде $A=\left(V_{1} \sqcap V_{2}\right) \sqcap A \sqcap\left(U_{2} \sqcap U_{1}\right)$, где $V_{1} \sqcap V_{2}$ и $U_{2} \sqcap U_{1}$ - некоторые 
квадратные матрицы. Известно, что квадратные булевы матрицы порождают конечные циклические полугруппы. Это означает, что всегда существуют такие степени булевых матриц $V=\left(V_{1} \sqcap V_{2}\right)^{k_{0}}$ и $U=\left(U_{2} \sqcap U_{1}\right)^{s_{0}}$, которые являются идемпотентами, то есть $V=V^{2}$ и $U=U^{2}$. Например, в качестве $k_{0}$ и $s_{0}$ можно взять произведения индексов и периодов каждой из циклических полугрупп этих матриц соответственно (см. раздел 1.6 в [15]). Умножая $k_{0} s_{0}$ раз обе части равенства $A=\left(V_{1} \sqcap V_{2}\right) \sqcap A \sqcap\left(U_{2} \sqcap U_{1}\right)$ слева и справа на $V_{1} \sqcap V_{2}$ и $U_{2} \sqcap U_{1}$ соответственно, получаем, что

$$
A=\left(V_{1} \sqcap V_{2}\right) \sqcap A \sqcap\left(U_{2} \sqcap U_{1}\right)=\ldots=\left(V_{1} \sqcap V_{2}\right)^{k_{0} s_{0}} \sqcap A \sqcap\left(U_{2} \sqcap U_{1}\right)^{k_{0} s_{0}}
$$

или

$$
A=V^{s_{0}} \sqcap A \sqcap U^{k_{0}}=V \sqcap A \sqcap U
$$

в силу идемпотентности элементов $V$ и $U$. Тогда

$$
V \sqcap A=V^{2} \sqcap A \sqcap U=V \sqcap A \sqcap U=A=V \sqcap A \sqcap U^{2}=A \sqcap U .
$$

Следовательно,

$$
\begin{aligned}
A & =V \sqcap A \sqcap U=V \sqcap V \sqcap A=V \sqcap A=\left(V_{1} \sqcap V_{2}\right)^{k_{0}} \sqcap A \\
& =\left(V_{1} \sqcap V_{2}\right)^{k_{0}-1} \sqcap\left(V_{1} \sqcap V_{2}\right) \sqcap A=\left(\left(V_{1} \sqcap V_{2}\right)^{k_{0}-1} \sqcap V_{1}\right) \sqcap\left(V_{2} \sqcap A\right) .
\end{aligned}
$$

По предложению 2 это дает включение строчных пространств $R(A) \subseteq R\left(V_{2} \sqcap A\right)$. С другой стороны, $R(A) \supseteq R\left(V_{2} \sqcap A\right)$. Последнее означает, что $\left(A, V_{2} \sqcap A\right) \in \varepsilon_{R}$. Аналогично можно показать, что $\left(A, A \sqcap U_{2}\right) \in \varepsilon_{C}$. Учитывая сохранение отношений $\varepsilon_{R}$ и $\varepsilon_{C}$ при правых и, соответственно, левых умножениях (предложение 4) и то, что $B=V_{2} \sqcap A \sqcap U_{2}$, получаем, чTO

$$
\left(V_{2} \sqcap A, V_{2} \sqcap A \sqcap U_{2}\right)=\left(V_{2} \sqcap A, B\right)=\left(B, V_{2} \sqcap A\right) \in \varepsilon_{C} .
$$

Последнее вместе с полученным выше условием $\left(A, V_{2} \sqcap A\right) \in \varepsilon_{R}$ приводит к включению $(A, B) \in \varepsilon_{D}$. Это означает равенство эквивалентностей $\varepsilon_{D}=\varepsilon_{J}$ в силу произвольности выбора пары матриц $(A, B) \in \varepsilon_{J}$.

Следующее утверждение указывает на то, что необходимым признаком принадлежности матриц одному классу перечисленных выше отношений эквивалентности на группоиде М(В) является равенство рангов факторизации.

Теорема 1. Ранги факторизации всех матриц, находящихся в одном єЈ-классе частичного группоида всевозможных булевых матрии М(B), равны.

Доказательство. Действительно, для любой матрицы $B \in \varepsilon_{J}\langle A\rangle$, где $A \in \mathbf{B}_{m \times n}$, найдутся такие матрицы $U_{1}, U_{2}, V_{1}, V_{2}$, что $A=V_{1} \sqcap B \sqcap U_{1}$ и $B=V_{2} \sqcap A \sqcap U_{2}$. С учетом предложения 6 это означает, что $\operatorname{rank}_{f} A \leqslant \operatorname{rank}_{f} B$ и $\operatorname{rank}_{f} B \leqslant \operatorname{rank}_{f} A$, то есть $\operatorname{rank}_{f} A=\operatorname{rank}_{f} B$.

Из соотношений $\varepsilon_{H} \subseteq \varepsilon_{R(C)} \subseteq \varepsilon_{C} \cdot \varepsilon_{R}=\varepsilon_{R} \cdot \varepsilon_{C}=\varepsilon_{D}=\varepsilon_{J}$ следует, что все матрицы, находящиеся в одном классе перечисленных здесь эквивалентностей, обладают одним и тем же рангом факторизации. Таким образом, каждый столбец или строку матрицы из данного класса можно представлять в виде линейных комбинаций каких-то, не обязательно принадлежащих самой матрице, столбцов или строк, число которых нельзя сделать меньше, чем ранг факторизации данной матрицы. 
Предложение 10. Для любых двух матриц $A$ и $B$ из одного $\varepsilon_{J}$-класса справедливы равенства

$$
\operatorname{rank}_{C} A=\operatorname{rank}_{C} B, \quad \operatorname{rank}_{R} A=\operatorname{rank}_{R} B
$$

Доказательство. Предположим, что матрицы $A$ и $B$ принадлежат одному $\varepsilon_{J}$-классу, но $\varepsilon_{R}\langle A\rangle \neq \varepsilon_{R}\langle B\rangle$. Пусть, например, $\operatorname{dim} R(A)>\operatorname{dim} R(B)$. Тогда в классе $\varepsilon_{R}\langle B\rangle$ существует матрица $\tilde{B}$ размера $\operatorname{dim} R(B) \times s$, где $s-$ число столбцов матрицы $B$. Из предложения 8 следует, что класс $\varepsilon_{C}\langle\tilde{B}\rangle$ пересекается с классом $\varepsilon_{R}\langle A\rangle$. Матрицы такого пересечения должны иметь столько же строк, сколько у матрицы $\tilde{B}$, и число столбцов $t$, равное числу столбцов матрицы $A$. Это означает, что матрицы из пересечения должны быть размеpa $\operatorname{dim} R(B) \times t$. Но в классе $\varepsilon_{R}\langle A\rangle$ таких матриц нет, так как минимальное число их строк равно $\operatorname{dim} R(A)$. Получаем противоречие. Следовательно, $\operatorname{dim} R(A)=\operatorname{dim} R(B)$ или $\operatorname{rank}_{R} A=\operatorname{rank}_{R} B$.

Равенство $\operatorname{rank}_{C} A=\operatorname{rank}_{C} B$ для любых матриц $A$ и $B$ из одного $\varepsilon_{J}$-класса доказывается аналогично.

Предложение 8 показывает, что устройство каждого $\varepsilon_{J}$-класса группоида М(В) похоже на так называемую egg-box-картинку, строчки в которой, предположим, являются $\varepsilon_{R}$-классами, а $\varepsilon_{C}$-классы образуют столбцы. Отметим также, что $\varepsilon_{H}$-классы получаются пересечением строк со столбцами. Все матрицы $\varepsilon_{H}$-класса имеют одинаковые размеры, что сразу следует из предложения 3. Все матрицы в любой строчке $\varepsilon_{R}\langle A\rangle$ такой egg-box-картинки имеют одинаковое число столбцов, что и матрица $A$, поэтому можно расположить $\varepsilon_{H}$-классы такой строчки справа налево по убыванию числа строк матриц, принадлежащих этому классу $\varepsilon_{R}\langle A\rangle$, причем наименьшее число строк матриц, принадлежащих классу $\varepsilon_{R}\langle A\rangle$, равно $\operatorname{dim} R(A)$. Можно получить и аналогичное расположение $\varepsilon_{H}$-классов по убыванию числа столбцов матриц из класса $\varepsilon_{C}\langle A\rangle$ сверху вниз в столбцах egg-box-картинки. Наименьшее число столбцов матриц, принадлежащих классу $\varepsilon_{C}\langle A\rangle$, равно $\operatorname{dim} C(A)$. Так как для любой матрицы $A$ в $\varepsilon_{J}\langle A\rangle$-классе нет таких матриц, число строк или столбцов которых было бы меньше, чем $\operatorname{dim} R(A)$ или $\operatorname{dim} C(A)$ соответственно, то $\varepsilon_{H}$-класс матриц размеров $\operatorname{dim} R(A) \times \operatorname{dim} C(A)$ (или $\operatorname{rank}_{R} A \times \operatorname{rank}_{C} A$ ) будет расположен в нижнем левом углу построенной нами таким образом egg-box-картинки.

С другой стороны, в данном $\varepsilon_{J}\langle A\rangle$-классе есть матрицы любых конечных размеров, так как, по меньшей мере, столбцы (или строки) в таких матрицах могут повторяться, увеличивая их число, при этом столбцовые (или строчные) пространства матриц сохраняются.

Как мы видели, ранг факторизации, строчные и столбцовые ранги являются инвариантами для матриц из одного $\varepsilon_{J}$-класса.

\section{5. Определители и перманенты булевых матриц и некоторые их свойства}

Определение 8. Ориентированные полуперманенты $\stackrel{+}{\nabla} A$ и $\bar{\nabla} A$ (или компоненты бидетерминанта) $n \times n$ матрицы $A, n \geqslant 2$, с элементами из произвольной булевой алгебры 
определяются равенствами

$$
\begin{aligned}
& \stackrel{+}{\nabla} A=\bigcup_{\left(\alpha_{1}, \ldots, \alpha_{n}\right) \in \stackrel{+}{P}}\left(a_{1}^{\alpha_{1}} \cap \ldots \cap a_{n}^{\alpha_{n}}\right), \\
& \bar{\nabla} A=\bigcup_{\left(\alpha_{1}, \ldots, \alpha_{n}\right) \in \bar{P}}\left(a_{1}^{\alpha_{1}} \cap \ldots \cap a_{n}^{\alpha_{n}}\right),
\end{aligned}
$$

где $a_{j}^{i}$ - элементы матрицы $A$, а через $\stackrel{+}{P}$ и $\bar{P}$ обозначаются четные и нечетные $n$-перестановки верхних индексов соответственно. Ориентированные полуперманенты позволяют ввести перманент $\operatorname{Per} A=\stackrel{+}{\nabla} A \cup \bar{\nabla} A$. Определителем булевых матриц называют симметрическую разность полуперманентов

$$
\operatorname{Det} A=(\stackrel{+}{\nabla} A \backslash \bar{\nabla} A) \cup(\bar{\nabla} A \backslash \stackrel{+}{\nabla} A)
$$

Известны такие свойства определителя, как сохранение при транспонировании, он не изменяется при перестановке строк, если строки или столбцы линейно зависимы, то определитель равен нулю (обратное не выполняется в общем случае). Для ориентированных полуперманентов $\stackrel{+}{\nabla} A, \bar{\nabla} A$ и перманента $\operatorname{Per} A$ справедливы формулы Лапласа, дающие разложения по элементам строк или столбцов любой квадратной матрицы $A$ над произвольным коммутативным полукольцом. Для определителя $\operatorname{Det} A$ формулы Лапласа не выполняются в особых случаях, см. [5].

Вывод следующих формул, приведенных в предложении 11 и являющихся аналогами формул Коши-Бине, можно найти в [7, 9]. Можно воспользоваться также рассуждениями, приведенными в [3, 4] и в разделе 19 монографии [17] для ориентированных полуперманентов (бидетерминантов) матриц над коммутативным полукольцом. Здесь они приводятся без доказательства.

Предложение 11. Для произведения любых квадратных булевых матрии одного размера $\operatorname{Per}(A \sqcap B) \supseteq \operatorname{Per} A \cap \operatorname{Per} B, \operatorname{Det}(A \sqcap B) \subseteq \operatorname{Det} A \cap \operatorname{Det} B$.

Следующее утверждение обобщает свойство определителя булевой матрицы обращаться в ноль в случае, когда по крайней мере один столбец (строка) этой матрицы является линейной комбинацией остальных.

Предложение 12. Пусть для некоторой квадратной матрищьы $A \in \mathbf{B}_{n \times n}$ существует набор, не обязательно выбираемых из матриць $A$ столбияов $B_{1}, \ldots, B_{k}$, принадлежащих $\mathbf{B}_{n \times 1}$ такой, что любой столбеи $A_{1}, \ldots, A_{n} \in \mathbf{B}_{n \times 1}$ матрицуы $А$ является их линейной комбинацией. Тогда если $k<n$, то $\operatorname{Det} A=0$. Аналогичное утверждение справедливо $u$ для строк.

Доказательство. Вначале покажем, что для полуперманентов матрицы $A \in \mathbf{B}_{n \times n}, j$-й столбец которой является линейной комбинацией $A_{j}=\bigcup_{t=1}^{k}\left(\lambda^{t} \cap B_{t}\right)$ множества столб- 
цов $\left\{B_{j}=\left(b_{j}^{1}, \ldots, b_{j}^{n}\right)^{T}, j=1, \ldots, k\right.$, выполняется равенство

$$
\begin{aligned}
& \pm\left(\begin{array}{lllll}
\ldots & a_{j-1}^{1} & \bigcup_{t=1}^{k}\left(\lambda^{t} \cap b_{t}^{1}\right) & a_{j+1}^{1} & \ldots \\
\ldots \ldots \ldots \ldots \ldots \ldots \ldots \ldots \ldots \ldots \ldots \ldots \ldots \ldots & \ldots \ldots \\
\ldots & a_{j-1}^{n} & \bigcup_{t=1}^{k}\left(\lambda^{t} \cap b_{t}^{n}\right) & a_{j+1}^{n} & \ldots
\end{array}\right)
\end{aligned}
$$

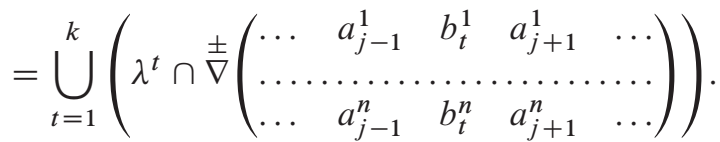

Здесь знаки +, - полуперманентов выбираются либо одновременно верхние, либо одновременно нижние. Это равенство показывает, что полуперманенты обладают свойством линейности по столбцу (по строке). Действительно,

$$
\begin{aligned}
& \pm\left(\begin{array}{lllll}
\ldots & a_{j-1}^{1} & \bigcup_{t=1}^{k}\left(\lambda^{t} \cap b_{t}^{1}\right) & a_{j+1}^{1} & \ldots \\
\ldots \ldots \ldots \ldots \ldots \ldots \ldots \ldots \ldots \ldots \ldots \ldots \ldots \ldots \ldots & \ldots \ldots \ldots \\
\ldots & a_{j-1}^{n} & \bigcup_{t=1}^{k}\left(\lambda^{t} \cap b_{t}^{n}\right) & a_{j+1}^{n} & \ldots
\end{array}\right) \\
& =\bigcup_{\left(\alpha_{1}, \ldots, \alpha_{n}\right) \in \stackrel{ \pm}{P}}\left(a_{1}^{\alpha_{1}} \cap \ldots \cap \bigcup_{t=1}^{k}\left(\lambda^{t} \cap b_{t}^{\alpha_{j}} \cap \ldots \cap a_{n}^{\alpha_{n}}\right)\right) \\
& =\bigcup \bigcup_{\left(\alpha_{1}, \ldots, \alpha_{n}\right) \in P^{ \pm}}^{k}\left(a_{1}^{\alpha_{1}} \cap \ldots \cap\left(\lambda^{t} \cap b_{t}^{\alpha_{j}}\right) \cap \ldots \cap a_{n}^{\alpha_{n}}\right) \\
& =\bigcup_{\left(\alpha_{1}, \ldots, \alpha_{n}\right) \in \pm} \bigcup_{t=1}^{k}\left(\lambda^{t} \cap\left(a_{1}^{\alpha_{1}} \cap \ldots \cap b_{t}^{\alpha_{j}} \cap \ldots \cap a_{n}^{\alpha_{n}}\right)\right. \\
& =\bigcup_{t=1}^{k}\left(\lambda^{t} \cap \bigcup_{\left(\alpha_{1}, \ldots, \alpha_{n}\right) \in \pm}{ }_{P}\left(a_{1}^{\alpha_{1}} \cap \ldots \cap b_{t}^{\alpha_{j}} \cap \ldots \cap a_{n}^{\alpha_{n}}\right)\right) \\
& =\bigcup_{t=1}^{k}\left(\lambda^{t} \cap \pm\left(\begin{array}{ccccc}
\ldots & a_{j-1}^{1} & b_{t}^{1} & a_{j+1}^{1} & \ldots \\
\ldots \ldots & \ldots \ldots \ldots \ldots \ldots \ldots \ldots \ldots \\
\ldots & a_{j-1}^{n} & b_{t}^{n} & a_{j+1}^{n} & \ldots
\end{array}\right)\right) \text {. }
\end{aligned}
$$

Для произвольных элементов $\lambda^{t}, c_{t}, d_{t}, t=1, \ldots, k$, некоторой булевой алгебры рассмотрим выражение $\left(\bigcup_{t=1}^{k}\left(\lambda^{t} \cap c_{t}\right)\right) \backslash\left(\bigcup_{s=1}^{k}\left(\lambda^{s} \cap d_{s}\right)\right)$ и преобразуем его следующим образом:

$$
\begin{aligned}
&\left(\left(\lambda^{1} \cap c_{1}\right) \cup\left(\lambda^{2} \cap c_{2}\right) \cup \ldots \cup\left(\lambda^{k} \cap c_{k}\right)\right) \backslash\left(\left(\lambda^{1} \cap d_{1}\right) \cup\left(\lambda^{2} \cap d_{2}\right) \cup \ldots \cup\left(\lambda^{k} \cap d_{k}\right)\right) \\
&=\left\{\left[\left(\lambda^{1} \cap c_{1}\right) \backslash\left(\lambda^{1} \cap d_{1}\right)\right] \backslash \bigcup_{t_{1} \neq 1}\left(\lambda^{t_{1}} \cap d_{t_{1}}\right)\right\} \cup\left\{\left[\left(\lambda^{2} \cap c_{2}\right) \backslash\left(\lambda^{2} \cap d_{2}\right)\right] \backslash \bigcup_{t_{2} \neq 2}\left(\lambda^{t_{2}} \cap d_{t_{2}}\right)\right\} \\
& \cup \ldots \cup\left\{\left[\left(\lambda^{k} \cap c_{k}\right) \backslash\left(\lambda^{k} \cap d_{k}\right)\right] \backslash \bigcup_{t_{k} \neq k}\left(\lambda_{t_{k}} \cap d_{t_{k}}\right)\right\} .
\end{aligned}
$$


Тогда

$$
\begin{aligned}
& \left(\left(\lambda^{1} \cap c_{1}\right) \cup\left(\lambda^{2} \cap c_{2}\right) \cup \ldots \cup\left(\lambda^{k} \cap c_{k}\right)\right) \backslash\left(\left(\lambda^{1} \cap d_{1}\right) \cup\left(\lambda^{2} \cap d_{2}\right) \cup \ldots \cup\left(\lambda^{k} \cap d_{k}\right)\right) \\
& \subseteq\left(\left(\lambda^{1} \cap c_{1}\right) \backslash\left(\lambda^{1} \cap d_{1}\right)\right) \cup\left(\left(\lambda^{2} \cap c_{2}\right) \backslash\left(\lambda^{2} \cap d_{2}\right)\right) \cup \ldots \cup\left(\left(\lambda^{k} \cap c_{k}\right) \backslash\left(\lambda^{k} \cap d_{k}\right)\right) .
\end{aligned}
$$

Остается заметить, что

$$
\left(\lambda^{t} \cap c_{t}\right) \backslash\left(\lambda^{t} \cap d_{t}\right)=\lambda^{t} \cap\left(c_{t} \backslash d_{t}\right)
$$

для всех $t$. Получаем, что

$$
\bigcup_{t=1}^{k}\left(\lambda^{t} \cap c_{t}\right) \backslash \bigcup_{s=1}^{k}\left(\lambda^{s} \cap d_{s}\right) \subseteq \bigcup_{t=1}^{k}\left(\lambda^{t} \cap\left(c_{t} \backslash d_{t}\right)\right) .
$$

Воспользовавшись предыдущими формулами, получаем включение

$$
\begin{aligned}
& +\stackrel{+}{\nabla}\left(\begin{array}{l}
\ldots a_{j-1}^{1} \bigcup_{t=1}^{k}\left(\lambda^{t} \cap b_{t}^{1}\right) a_{j+1}^{1} \ldots \\
\ldots \ldots \ldots \ldots \ldots \ldots \ldots \ldots \ldots \ldots \ldots \\
\ldots a_{j-1}^{n} \bigcup_{t=1}^{k}\left(\lambda^{t} \cap b_{t}^{n}\right) a_{j+1}^{n} \ldots
\end{array}\right) \backslash \bar{\nabla}\left(\begin{array}{l}
\ldots a_{j-1}^{1} \bigcup_{t=1}^{k}\left(\lambda^{t} \cap b_{t}^{1}\right) a_{j+1}^{1} \ldots \\
\ldots \ldots \ldots \ldots \ldots \ldots \ldots \ldots \ldots \ldots \\
\ldots a_{j-1}^{n} \bigcup_{t=1}^{k}\left(\lambda^{t} \cap b_{t}^{n}\right) a_{j+1}^{n} \ldots
\end{array}\right)
\end{aligned}
$$

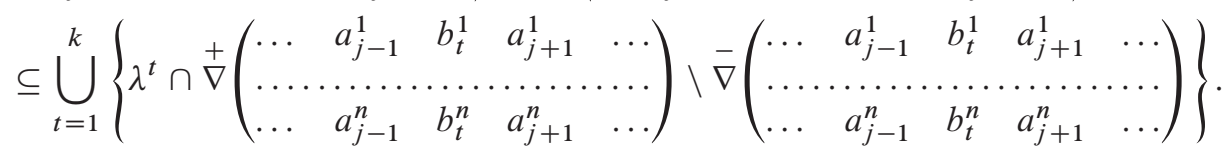

Аналогичные рассуждения можно провести и для разности $\bar{A} \backslash \stackrel{+}{A}$. Это позволяет получить следующее включение для определителя:

$$
\begin{aligned}
& \operatorname{Det}\left(\begin{array}{ccccc}
\ldots & a_{j-1}^{1} & \bigcup_{t=1}^{k}\left(\lambda^{t} \cap b_{t}^{1}\right) & a_{j+1}^{1} & \ldots \\
\ldots \ldots \ldots \ldots \ldots \ldots \ldots \ldots \ldots \ldots \ldots \ldots \ldots \ldots & \ldots \ldots \ldots \\
\ldots & a_{j-1}^{n} & \bigcup_{t=1}^{k}\left(\lambda^{t} \cap b_{t}^{n}\right) & a_{j+1}^{n} & \ldots
\end{array}\right) \\
& \subseteq \bigcup_{t=1}^{k}\left\{\lambda^{t} \cap \operatorname{Det}\left(\begin{array}{ccccc}
\ldots & a_{j-1}^{1} & b_{t}^{1} & a_{j+1}^{1} & \ldots \\
\ldots \ldots & \ldots \ldots \ldots \ldots \ldots \ldots \ldots \ldots \\
\ldots & a_{j-1}^{n} & b_{t}^{n} & a_{j+1}^{n} & \ldots
\end{array}\right)\right\} .
\end{aligned}
$$

Учитывая, что эту цепочку включений можно продолжить, применяя аналогичные рассуждения к каждому столбцу матрицы $A$, получим, что $\operatorname{Det} A$ содержится в некоторой линейной комбинации определителей квадратных матриц, построенных из столбцов $B_{1}, \ldots B_{k}$. Так как $k<n$, определители таких матриц содержат одинаковые столбцы и поэтому равны нулю. Следовательно, $\operatorname{Det} A=0$.

\section{6. Минорные и перманентные ранги булевых матриц}

Из элементов некоторой булевой матрицы $A \in \mathbf{B}_{m \times n}$, расположенных на пересечении некоторых $k$ столбцов с номерами $j_{1}<\ldots<j_{k}$ и строк с номерами $i_{1}<\ldots<i_{k}$ образуем квадратную булеву матрицу $M_{j_{1}, \ldots, j_{k}}^{i_{1}, \ldots, i_{k}}$. Понятно, что порядок $k$ этой матрицы ограничивается неравенством $1 \leqslant k \leqslant \min (m, n)$. Назовем эту матрицу $k$-блоком матрицы $A$. Для дальнейших рассуждений неважно, из каких строк и столбцов образован некоторый $k$-блок, поэтому в его обозначении номера строк и столбцов будут опускаться. Например, $M_{k}$ будет обозначать некоторый $k$-блок. 
Определение 9. Минорным рангом ненулевой матрицы назовем натуральное число $k$, $1 \leqslant k \leqslant \min (m, n)$, удовлетворяющее следующим двум условиям.

(1) У матрицы $A$ существует квадратный блок $M_{k}$ порядка $k$, определитель которого отличен от нуля;

(2) Если у матрицы существуют $(k+1)$-блоки, то их определители равны нулю.

Ранг нулевой матрицы считается равным нулю.

Если минорный ранг булевой матрицы $A$ равен $k$, то любой ее $k$-блок с отличным от нуля определителем назовем ранговым блоком. Столбцы (строки) матрицы $A$, которые участвуют в образовании рангового блока, назовем ранговыми столбцами (строками) соответственно.

Предложение 13. Если минорный ранг матрицыь $A$ равен $k$, то определитель всех ее блоков порядка $k+1$ и выше, если они существуют, равны нулю.

Доказательство. Непосредственно из определения минорного ранга следует, что ориентированные полуперманенты любого квадратного $(k+1)$-блока матрицы $A$ совпадают, то есть

$$
\stackrel{+}{\nabla} M_{k+1}=\bar{\nabla} M_{k+1}
$$

Возьмем теперь блок порядка $(k+2)$ и разложим его ориентированные полуперманенты по элементам его любого столбца или строки (см. [5]). Коэффициенты в этом разложении являются соответствующими ориентированными полуперманентами блоков матрицы $A$, порядка $k+1$, и следовательно, являются равными. Значит, ориентированные полуперманенты блоков порядка $k+2$ тоже равны. Таким образом, все определители $(k+2)$-блоков, если они существуют, равны нулю. По этим же причинам определители блоков и более высокого порядка, если они существуют, являются нулевыми.

Таким образом, ранг матрицы $A$ - это наибольший порядок квадратного блока, определитель которого отличен от нуля. Если такой ранг матрицы $A$ равен $k$, то пишем $\operatorname{rank} A=k$.

Замечание 1. Если у матрицы $A$ перманенты всех ее блоков порядка $k+1$ равны 0 , то перманенты всех ее блоков порядка $k+2$ и выше, если они существуют, тоже равны 0. Это позволяет ввести следующее понятие.

Определение 10. Перманентным рангом назовем наибольший порядок $k$ квадратичного блока, перманент которого отличен от нуля. Перманентный ранг нулевой матрицы считается равным нулю. Введем обозначение $k=\operatorname{rank}_{\mathrm{Per}} A$.

В теории матриц аналогичный ранг можно встретить под названиями граничного ранга, терм-ранга или ранга покрытия (см., например, $[18,19])$.

Очевидно, что из включения $\operatorname{Det} A \subseteq \operatorname{Per} A$, верного для любой матрицы $A$ с элементами из булевой алгебры, вытекает следующее утверждение.

Теорема 2. Для любой матрицьь с элементами из булевой алгебры выполняется неравенство

$\operatorname{rank}_{\mathrm{Per}} A \geqslant \operatorname{rank} A$. 
Пример 3. Для булевой матрицы

$$
A=\left(\begin{array}{ccc}
{[0 ; 1]} & {[0 ; 2]} & {[0 ; 3]} \\
{[-1 ; 0]} & \varnothing & {[0 ; 2]} \\
{[0 ; 1]} & {[0 ; 3]} & {[-1 ; 2]}
\end{array}\right)
$$

составленной из подмножеств действительной числовой прямой, минорный ранг равен 2 , а перманентный ранг равен 3, так как

$$
\stackrel{+}{\nabla} A=\bar{\nabla} A=[0 ; 1]
$$

Замечание 2. Перманентный ранг булевой матрицы может быть равен, больше или меньше строчного или столбцового ранга. Так, для булевой матрицы

$$
A=\left(\begin{array}{llll}
1 & 0 & 1 & 1 \\
1 & 1 & 0 & 0 \\
0 & 1 & 1 & 0
\end{array}\right)
$$

перманентный ранг равен 3, а столбцовый ранг равен 4 (см. пример 3.2 в разделе 3 в [10]). Ниже будет дан пример 6 матрицы, для которой выполняется противоположное неравенство $\operatorname{rank}_{\mathrm{Per}} A>\operatorname{rank}_{C(R)} A$.

\section{7. Ранги и определители матриц частичного группоида булевых матриц}

Рассмотрим теперь свойства элементов одного $\varepsilon J$-класса частичного группоида всех булевых матриц в контексте определителей и минорных рангов. Прежде всего покажем, что определители дают булевозначный инвариант для матриц из одного $\varepsilon_{J}$-класса.

Теорема 3. Определители квадратных матриц одного и того же размера равны, если эти матрицы находятся в одном $\varepsilon_{J}$-классе частичного группоида $\mathbf{M ( B ) ~ в с е в о з м о ж н ы х ~}$ булевых матриц конечного размера.

Доказательство. Для квадратных матриц $A$ и $B$ одного размера $r \times r$, находящихся в одном $\varepsilon_{J}$-классе, существуют такие квадратные матрицы $U_{1}, U_{2}, V_{1}, V_{2} \in \mathbf{B}_{r \times r}$, что

$$
A=V_{1} \sqcap B \sqcap U_{1}, \quad B=V_{2} \sqcap A \sqcap U_{2} .
$$

С учетом формулы предложения 11 , это означает, что

Det $A \subseteq \operatorname{Det} B \cap \operatorname{Det} V_{1} \cap \operatorname{Det} U_{1} \subset \operatorname{Det} B$,

Det $B \subseteq \operatorname{Det} A \cap \operatorname{Det} V_{2} \cap \operatorname{Det} U_{2} \subset \operatorname{Det} A$.

Поэтому

$$
\operatorname{Det} A=\operatorname{Det} B \text {. }
$$

Предложение 14. Если $A-$ квадратная $n \times n$ матрица с ненулевым определителем, то факторизационный ранг такой матрищы равен ее минорному рангу и равен $n$. 
Доказательство. Пусть некоторая квадратная матрица $A \in \mathbf{B}_{n \times n}$ имеет отличный от нуля определитель. Предположим, что ранг факторизации равен $\operatorname{rank}_{f} A=k$. По определению ранга факторизации и предложению 2 это означает, что существует набор столбцов $B_{1}, B_{2}, \ldots, B_{k} \in \mathbf{B}_{n \times 1}$, для которого $C(A) \subseteq S\left(\left\{B_{1}, B_{2}, \ldots, B_{k}\right\}\right)$. Учитывая предложение 12 , получаем, что $k \geqslant n$. С другой стороны, $\operatorname{rank}_{f} A \leqslant n$, так как всякую матрицу можно представить произведением с единичной матрицей $A=A \sqcap E$. Получаем, что для квадратной $n \times n$ матрицы с ненулевым определителем всегда $\operatorname{rank}_{f} A=n$.

Теорема 4. Минорный ранг булевой матрицьы не превосходит ее факторизационный ранг. Для любой матриць А справедливы неравенства

$$
\min \left(\operatorname{rank}_{R} A, \operatorname{rank} C A\right) \geqslant \operatorname{rank}_{f} A \geqslant \operatorname{rank} A .
$$

Доказательство. Если минорный ранг матрицы $A$ равен $\operatorname{rank} A=k$, то по предложению 12 число независимых столбцов, участвующих в образовании ранговых столбцов матрицы $A$, должно быть больше или равно $k$. Поэтому факторизационный ранг матрицы $A$ должен быть больше или равен ее минорному рангу. Учитывая предложение 5 , получаем, что

$$
\min \left(\operatorname{rank}_{R} A, \operatorname{rank}_{C} A\right) \geqslant \operatorname{rank}_{f} A \geqslant \operatorname{rank} A .
$$

Ниже приведены примеры вычислений рангов булевых матриц.

Пример 4. Для матрицы

$$
A=\left(\begin{array}{ccc}
{[0 ; 1]} & {[0 ; 2]} & {[0 ; 3]} \\
{[-1 ; 0]} & \varnothing & {[0 ; 2]} \\
{[0 ; 1]} & {[0 ; 3]} & {[-1 ; 2]}
\end{array}\right)
$$

с элементами из булевой алгебры всех подмножеств действительной числовой прямой

$$
\operatorname{Per} A=\stackrel{+}{\nabla} A=\bar{\nabla} A=[0 ; 1], \quad \operatorname{Det} A=0 .
$$

Поэтому

$$
\operatorname{rank}_{\text {Per }} A=3 \geqslant \operatorname{rank} A=2 .
$$

Так как

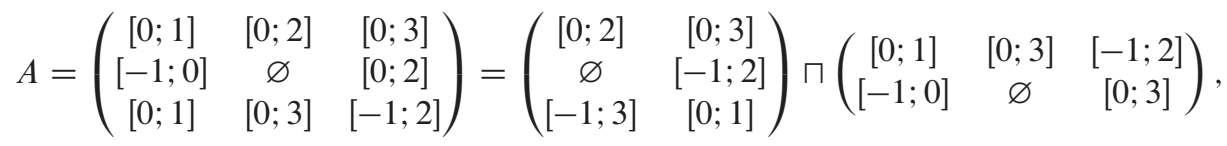

справедливы равенства

$$
\operatorname{rank}_{f} A=\operatorname{rank} A=2 .
$$

Пример 5. Справедливо разложение в произведение

$$
A=\left(\begin{array}{llll}
1 & 1 & 0 & 0 \\
1 & 1 & 1 & 0 \\
0 & 1 & 1 & 1
\end{array}\right)=\left(\begin{array}{lll}
1 & 0 & 0 \\
1 & 1 & 0 \\
0 & 1 & 1 \\
0 & 0 & 1
\end{array}\right) \sqcap\left(\begin{array}{llll}
1 & 1 & 0 & 0 \\
0 & 1 & 1 & 0 \\
0 & 0 & 1 & 1
\end{array}\right),
$$


что позволяет получить равенство

$$
\operatorname{rank}_{f} A=\operatorname{rank} A=3 .
$$

Матрица

$$
B=\left(\begin{array}{llll}
1 & 1 & 0 & 0 \\
0 & 1 & 1 & 0 \\
0 & 0 & 1 & 1 \\
1 & 0 & 0 & 1
\end{array}\right)
$$

имеет ранги

$$
4=\operatorname{rank}_{f} B>\operatorname{rank} B=3 .
$$

Пример 6. Рассмотренная ранее в примере 2 матрица

$$
A=\left(\begin{array}{lll}
(11100) & (01100) & (00000) \\
(00111) & (00110) & (00001) \\
(11111) & (01110) & (00001)
\end{array}\right)
$$

над булевой алгеброй $\mathbf{B}_{2}^{5}$ имеет ранги

$$
\operatorname{rank}_{C} A=\operatorname{rank}_{R} A=\operatorname{rank}_{j} A=\operatorname{rank} A=1,
$$

а ее перманентный ранг равен

$$
\operatorname{rank}_{\text {Per }} A=2 \text {. }
$$

Следующее утверждение показывает, в частности, что вместе с факторизационным рангом минорные ранги также характеризуют каждый $\varepsilon_{J}$-класс группоида всех булевых матриц М(B).

Теорема 5. Минорный ранг произведения любых булевых матрии не превосходит минорных рангов сомножителей. Таким образом, минорные ранги всех матриц, находящихся 8

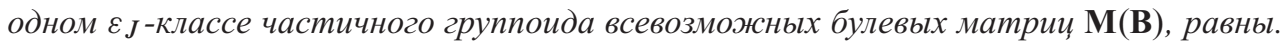

Доказательство. Пусть минорный ранг некоторой матрицы $A$ равен $k$. Рассмотрим какойлибо $(k+1)$-блок матрицы $A \sqcap B$, где $B-$ произвольная булева матрица. Пусть он расположен на строчках с номерами $i_{1}, \ldots, i_{k+1}$. Каждый столбец такого $(k+1)$-блока получается как линейная комбинация столбцов соответствующих (то есть с теми же номерами $i_{1}, \ldots, i_{k+1}$ строк) $(k+1)$-блоков матрицы $A$. Тогда, с учетом формулы $(1)$, заключаем, что определитель такого $(k+1)$-блока содержится в линейной комбинации определителей некоторых $(k+1)$-блоков матрицы $A$. Так как определители всевозможных $(k+1)$-блоков матрицы $A$ равны нулю, определитель такого произвольно выбранного $(k+1)$-блока матрицы $A \sqcap B$ тоже равен нулю. Следовательно, минорный ранг матрицы $A \sqcap B$ не может превосходить $\operatorname{rank} A=k$.

Тогда, очевидно, минорные ранги матриц, находящихся в одном $\varepsilon_{J}$-классе частичного группоида М(B), равны.

Теорема 6. В некотором $\varepsilon_{J}$-классе существует квадратная $n \times n$ матрица с ненулевым определителем тогда и только тогда, когда столбцовый, строчный, факторизационный и минорный ранги любой матрищы такого $\varepsilon_{J}$-класса равны между собой и равны $n$. Все матрицы того же размера $n \times n$ имеют равные определители, а определители

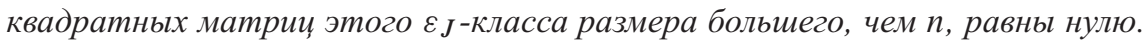


Доказательство. Если в некотором $\varepsilon_{J}$-классе найдется $n \times n$ матрица $A$ с ненулевым определителем, то, во-первых, ее размер не меньше наименьшего возможного размера $\operatorname{rank}_{R} A \times \operatorname{rank}_{C} A$, то есть

$$
n \geqslant \max \left(\operatorname{rank}_{R} A, \operatorname{rank}_{C} A\right),
$$

во-вторых, ее факторизационный ранг есть $n$ (предложение 14) и верно неравенство

$$
\min \left(\operatorname{rank}_{R} A, \operatorname{rank}_{C} A\right) \geqslant \operatorname{rank}_{f} A=n
$$

Получаем, что

$$
\operatorname{rank}_{R} A=\operatorname{rank}_{C} A=\operatorname{rank}_{f} A=\operatorname{rank} A=n .
$$

Обратно, если

$$
\operatorname{rank}_{R} A=\operatorname{rank}_{C} A=\operatorname{rank}_{f} A=\operatorname{rank} A=n
$$

для некоторой матрицы $A$, то в классе $\varepsilon_{J}\langle A\rangle$ существует квадратная матрица $B$ наименьшего возможного размера $\operatorname{rank}_{R} A \times \operatorname{rank}_{C} A=n \times n$. Кроме того, учитывая утверждения теорем 1 и 5, получаем, что

$$
\operatorname{rank}_{R} B=\operatorname{rank}_{C} B=\operatorname{rank}_{f} B=\operatorname{rank} B=n .
$$

Следовательно, Det $B \neq 0$.

Отметим также, что все матрицы того же размера $n \times n$ имеют равные определители, отличные от нуля (теорема 3). Определители большего размера квадратных матриц этого $\varepsilon_{J}$-класса равны нулю, так как минорный ранг таких матриц строго меньше их размера.

Существуют ли $\varepsilon J$-классы булевых матриц, все определители квадратных матриц которых равны нулю? Очевидно, что к таким классам следует отнести классы, в которых по крайней мере одна матрица обладает факторизационным рангом, строго большим, чем ее минорный ранг (матрица из примера 5 ). К таким $\varepsilon$-классам относятся прямоугольные классы, то есть классы, для которых столбцовые и строчные ранги различны $(\operatorname{dim} R(A) \neq \operatorname{dim} C(A))$, даже если факторизационный и минорный ранги совпадают. Действительно, предполагая существование в таком $\varepsilon_{J}$-классе матрицы, определитель которой не равен нулю, получаем противоречие с теоремой 6 .

\section{Список литературы}

1. Соколов О. Б., Применение булевых определителей к анализу логических многополюсников. Ученые записки Казанского госуниверситета (1963) 12, №6, 155-164.

2. Chesley D. S., Bevis J. H., Determinants for matrices over lattices. Proc. Royal Soc. Edinburgh (1969) 68, 138-144.

3. Reutenauer C., Straubing H., Inversion of matrices over a commutative semiring. J. Algebra (1984) 88, 350-360.

4. Poplin P. L., Hartwig R. E., Determinantal identities over commutative semirings. Linear Algebra Appl. (2004) 387, 99-132.

5. Поплавский В. Б., О разложении определителей булевых матриц. Фундаментальная и прикладная математика (2007) 13, №4, 199-223. 
6. Poplavski V., On orientability and degeneration of Boolean binary relation on a finite set. In: Math. Logic in Asia. Proc. 9th Asian Logic Conf. World Scientific, Singapore, 2006, pp. 203-214.

7. Поплавский В. Б., Определители степеней булевых матриц. Чебышевский сборник (2004) 5, №3 (11), 98-111.

8. Поплавский В. Б., Объемы и определители степеней транзитивных и рефлексивных булевых отношений на конечных множествах. Известия Тульского госуниверситета. Серия Математика. Механика. Информатика (2004) 10, №1, 134-141.

9. Поплавский В. Б., Ориентированные определители произведения булевых матриц. В сб.: Математика. Механика, 6. Изд-во Саратовского унив., 2004, с. 111-114.

10. Beasley L. B., Guterman A. E., Rank inequalities over semirings. J. Korean Math. Soc. (2005) 42, 223-241.

11. Пшеницина О. А., Факторизационный и граничный ранг матричного объединения над полукольцом. Фундаментальная и прикладная математика (2004) 9, №3, 175-197.

12. Бисли Л. Б., Гутерман А. Э., Йи С.-Ч., LР-проблемы для ранговых неравенств над полукольцами: граничные ранги. Фундаментальная и прикладная математика (2004) 10, №2, 3-12.

13. Song Seok-Zun, Lee Sang-Gu, Column ranks and their preservers of general Boolean matrices. $J$. Korean Math. Soc. (1995) 32, 531-540.

14. Kim Ki Hang, Boolean matrix theory and applications. Marcel Dekker, New York, 1982.

15. Клиффорд А., Престон Г., Алгебраическая теория полугрупn, 1. Мир, Москва, 1985.

16. Лаллеман Ж., Полугруппы и комбинаторные приложения. Мир, Москва, 1985.

17. Golan J. S., Semirings and their applications. Kluwer, Dordrecht, 1999.

18. Сачков В. Н., Тараканов В. Е., Комбинаторика неотрицательных матриц. ТВП, Москва, 2000.

19. Минк Х., Перманентыл. Мир, Москва, 1982.

Статья поступила 10.01.2007. 